\title{
Propuesta de código ético para la asignatura de Gestión de la Calidad: comparativa interuniversitaria
}

\author{
Code of conduct proposal for the Quality management subject: \\ interuniversity comparison
}

\author{
Merce Bernardo (merce.bernardo@ub.edu) \\ Universitat de Barcelona (España) \\ Juan José Tarí (jj.tari@ua.es) \\ Universidad de Alicante (España) \\ http://dx.doi.org/10.12795/EDUCADE.2013.i04.08
}

RESUMEN: El marco europeo de educación superior ha supuesto un reciclaje en la metodología de enseñanza. Una de las características del tratado de Bolonia es la evaluación continua del alumnado, el cual es evaluado periódicamente para analizar el alcance de sus conocimientos y el desarrollo de sus competencias.

Del éxito de esta metodología depende la motivación y la satisfacción de los estudiantes, por ello, el primer objetivo de este artículo es analizar la satisfacción de los alumnos con la metodología de evaluación continua. El nuevo sistema de enseñanza-aprendizaje requiere más interacción entre profesores-alumnos y alumnos-alumnos. Esto aumenta la necesidad de unas normas de comportamiento en las clases. Por ello, nos planteamos como segundo objetivo elaborar un código de conducta para la asignatura de Gestión de la Calidad.

La metodología aplicada para conocer la satisfacción del alumnado ha sido un cuestionario sobre aspectos diversos de la asignatura, entre ellos la metodología de evaluación. El código ético es propuesto por los estudiantes como un ejercicio de la evaluación continua. El análisis se ha llevado a cabo en la Universidad de Alicante y la Universitat de Barcelona.

Los resultados muestran opiniones similares con relación a la metodología docente. Por ejemplo, el alumno muestra sus preferencias por la evaluación continua frente a la tradicional. De igual forma, los aspectos más destacables de los códigos éticos son: cumplir con la legislación vigente, respetar a las personas, sancionar los comportamientos inadecuados como copiar en los exámenes, la corrupción, el soborno, etc.

La propuesta de código ético que se presenta es para ambas universidades y para esta asignatura, pero tiene la voluntad de poderse implantar en otras asignaturas que deseen seguir esta práctica.

PALABRAS CLAVE: Evaluación continua, Gestión de la calidad, Código ético, Universidad.

ABSTRACT: The European framework for higher education meant recycling the teaching methodology. One characteristic of the Bologna Agreement is the students' continuous assessments, which are periodically evaluated to analyse the scope of their knowledge and competence development.

The success of this approach depends on the motivation and satisfaction of students; therefore, the first aim of this paper is to analyse the students' satisfaction with the continuous assessment methodology. New teaching-learning system requires more interaction between lecturersstudents and students-students.

Experiencia docente. Recibido: 30-05-13 - Versión revisada: 16-07-13, Aceptado: 05-09-13

Licencia Creative Commons BY NC ND · 2013 · Asociación Española de Contabilidad y Administración de Empresas - AECA 
This increases the need for standards of behavior in classes. Therefore, the second aim is to propose a code of conduct for the Quality Management subject.

The methodology applied to know the students' satisfaction is a questionnaire about different aspects of the subject, among them the evaluation methodology. The code of conduct is proposed by the student's as an exercise of the continuous assessment. The analysis has been done at the Universidad de Alicante and Universitat de Barcelona.

The results show similar opinions regarding the teaching methodology. For example, the students prefer the continuous assessment rather the traditional evaluation. Similarly, the most highlighted aspects of the codes of conduct are: legal compliance, respect people, punishing inadequate behaviours such as cheating, corruption, bribery, etc.

The code of conduct proposal presented is for both universities and for the specific subject, but it has the will to be implemented in other subjects wishing to follow this practice

KEYWORDS: Continuous assessment, Quality management, Code of conduct, University.

\section{INTRODUCCIÓN}

El nuevo marco europeo de educación superior ha supuesto un reciclaje en la metodología de enseñanza de muchas universidades españolas. Una de las características del tratado de Bolonia es la evaluación continua del alumnado, que es evaluado periódicamente para conocer el alcance de sus conocimientos y el desarrollo de sus competencias. Diversos autores han sugerido que la evaluación continua es un buen sistema para valorar la adquisición de competencias, ya que consiste en hacer un seguimiento individual del proceso de aprendizaje del alumnado, principal participante de su aprendizaje, maximizando las posibilidades de retroalimentación (Plaza et al., 2010). Aunque se han hecho avances importantes en la innovación docente (aprendizaje cooperativo, contrato de aprendizaje, etc.), según el informe del Ministerio de Educación y Ciencia del Gobierno español que incluye las "Propuestas para la renovación de la metodología docente en la Universidad" (Ministerio de Educación y Ciencia, 2006), en muchos casos las clases teóricas basadas en la clase magistral continúan siendo la principal herramienta para la docencia. En este contexto, la investigación en métodos de enseñanza-aprendizaje es un aspecto crucial (Martínez y Sauleda, 2004, 2005, Michavila y Pérez, 2007, Garandillas et al., 2011, Pascual et al., 2011).

En este proceso de enseñanza-aprendizaje, se está abandonando, de forma rápida, la metodología anterior centrada en la clase magistral en la que el profesor transmitía los contenidos (de Juana-Espinosa et al., 2010) y se evaluaba al alumnado en un único examen final. Además, el proceso de evaluación no se puede separar del de aprendizaje ya que la retroalimentación que recibe el estudiante en la evaluación le sirve de guía (de Juana-Espinosa et al., 2010). Por esta razón, en el contexto europeo, la evaluación necesita adquirir una dimensión superior, ya que su papel es el de medir la adquisición de las competencias definidas, mientras que contribuye al aprendizaje y desarrollo de las mismas (Sabater et al., 2012).

Esta metodología permite que los estudiantes tomen conciencia de su nivel de progreso, siendo una forma de aprendizaje continuo a lo largo del período de estudio. Por ello, se espera que una evaluación continua que periódicamente propone actividades ayudará a los estudiantes a asimilar las competencias y contenidos de cada tema progresivamente (de Juana-Espinosa et al., 2010). 
De este modo, las universidades españolas están introduciendo cambios en la metodología docente y en los procesos de evaluación para ajustarse al marco europeo. En este contexto, los estudiantes realizan más exámenes para evaluar las diferentes partes de la asignatura y presentan más proyectos, y la metodología cambia para reforzar las actividades prácticas en clase.

Todos estos cambios también significan el desarrollo de competencias de los estudiantes, que deben ser más activos y participativos en su proceso de aprendizaje (Ruiz y Castaño, 2008, Delgado y Oliver, 2006, González y Ramos, 2007, MontoroSanchez et al., 2012). Estos cambios han tenido efectos positivos en las actividades docentes de diversas universidades (Garandillas et al., 2011; González Pareja et al., 2004; Gras García et al., 2006, Claver Cortés et al., 2007; Sabater et al., 2012):

- Ayuda a los estudiantes a actualizar la asignatura posibilitando la preparación del examen con tiempo suficiente para superarlo y no dejarlo para el final.

- Proporciona una evaluación previa de los conocimientos adquiridos progresivamente.

- Permite obtener una nota adicional como premio por el trabajo hecho.

- La docencia en el aula es más entretenida con clases más interactivas y participativas.

- Hay un mayor número de oportunidades de aprobar la asignatura (a través de trabajos adicionales o convocatorias extraordinarias).

- El profesor muestra interés por las opiniones de los estudiantes, que permite una comunicación más fluida entre el profesor y los estudiantes.

En esta evaluación constante del alumnado, se ha pasado del control de calidad al final de la asignatura, con un examen final que aglutinaba toda la materia de la asignatura; a un aseguramiento de la calidad en el cual se intenta que el alumnado adquiera los conocimientos adecuados a través de una revisión constante de si esta adquisición se hace de forma correcta según los requisitos de la asignatura. En esta situación, y de acuerdo con los principios básicos de la gestión de la calidad (ISO, 2005), el objetivo principal de las organizaciones es que sus productos y/o servicios satisfagan a sus clientes y otros grupos de interés (EFQM, 2012), por lo que es necesario analizar las opiniones de los clientes para mejorar (ISO, 2005, ISO, 2009). En un contexto universitario, los principales clientes son los estudiantes, y escuchar sus necesidades y expectativas es un aspecto clave en el proceso de mejora de la calidad universitaria. Por ello, muchos profesores universitarios en España están desarrollando encuestas para poder conocer estas opiniones de sus estudiantes y poder mejorar así las asignaturas.

Además de esta satisfacción, se pretende o se debería pretender que la participación de los estudiantes sea máxima, propiciando un clima en el aula que promueva la interacción y participación activa del alumnado. Por ello debe garantizarse el respeto por todas las opiniones, sean de quien sean; así como actuar dentro de las normas establecidas. Esta situación no siempre es posible por razones diversas, ya sean de carácter intrínseco del alumnado (timidez, desinterés, etc.) o del profesor, o por motivos extrínsecos, como puede ser el aula o la diversidad geográfica del grupo. Para evitar que esto ocurra y que la docencia se desarrolle adecuadamente, algunas universidades han creado e implantado códigos éticos para garantizar el funcionamiento correcto de la institución (ver por ejemplo, UCA, 2005).

Relacionando ambos conceptos, a principios del año 2000 apareció la familia de estándares ISO 10000 sobre gestión de la calidad, pero focalizados en la satisfacción del cliente. Los cuatro estándares más destacados son: código de conducta (ISO, 
2007a), resolución interna de conflictos (ISO, 2004), resolución externa de conflictos (ISO, 2007b), y medida y seguimiento de la satisfacción del cliente (ISO, 2009).

\section{PROPÓSITO DEL TRABAJO}

Teniendo esto en cuenta, los objetivos de este artículo son dos. Primero, conocer la opinión del alumnado respecto al proceso de evaluación continua de la asignatura. Segundo, proponer un código ético para la asignatura de gestión de la calidad. En este sentido, en el temario se habla de las normas de aseguramiento de la calidad y se comentan las normas de la familia ISO 10000 sobre satisfacción de los clientes.

El primer objetivo se justifica porque conocer la satisfacción del alumnado es muy importante para poder mejorar de manera constante el proceso de aprendizaje. El segundo objetivo ha sido analizado desde el punto de vista de las empresas, en cuanto a diseño e implantación (ver por ejemplo, Adam y Rachman-Moore, 2004, Sethi et al., 2011), y desde el punto de vista académico (ver por ejemplo, Braxton y Bray, 2012), pero no se han detectado códigos de conducta diseñados por los propios alumnos como se presenta en este artículo. Implicar al alumnado en el diseño de este tipo de herramientas de gestión les puede ayudar en su futuro profesional y en la mejora de la convivencia dentro y fuera del aula porque les permitirá entender mejor los criterios de respecto en clase y, a su vez, conocer cómo se realiza un código ético.

\section{METODOLOGÍA}

El estudio se ha llevado a cabo en dos universidades diferentes, la Universidad de Alicante y la Universitat de Barcelona (para otra comparativa ver, por ejemplo, Castilla, 2011). La asignatura de Gestión de la Calidad en ambas universidades tiene como objetivo conocer el concepto de calidad y gestión de la calidad además de los métodos, sistemas, estándares y herramientas para implantar la cultura de la calidad en la empresa. En ambas universidades la asignatura de Gestión de la Calidad es optativa de cuarto curso del grado en Administración y Dirección de Empresas. Se ha escogido esta asignatura porqué muchas de las prácticas inspiradas en el marco europeo de educación superior ya se han implantado y sería más fácil aplicar la evaluación continua.

La metodología usada en la asignatura en la Universidad de Alicante está basada en una combinación de clases teóricas $(50 \%)$ y el estudio de casos de empresa $(50 \%)$ sobre los que los estudiantes tienen que debatir un conjunto de preguntas -para centrar el proceso de docencia-aprendizaje en el estudiante. Además, los estudiantes tienen que hacer dos exámenes test, presentar dos casos y un proyecto final. La evaluación resultante está basada en los dos exámenes test (50\%), un examen práctico hecho conjuntamente con cada test (10\%), dos estudios de casos (10\%) y un proyecto final (30\%).

La metodología usada en la asignatura en la Universitat de Barcelona es un examen final (50\%) y la evaluación continua (50\%). En esta última parte, el estudiante tiene 5 actividades evaluables, cada una al final de cada unidad, y cada actividad está compuesta por al menos dos tareas, por ejemplo, la actividad evaluable 1 consiste en un examen test y un estudio de caso. La nota final de la evaluación continua es la media de las 4 mejores notas obtenidas en las 5 actividades. Además, los estudiantes hacen pequeños ejercicios en clase, no evaluables, para: 1) aplicar la teoría en ejercicios prácticos, y 2) controlar la asistencia.

Para el primer objetivo, el alumnado cumplimentó a final del cuatrimestre una encuesta de satisfacción con la evaluación continua. En concreto, se recogieron 
datos cuantitativos a través de un cuestionario de preguntas cerradas medidas en una escala Likert de 1-5. El cuestionario incluyó cuatro partes:

- En la primera parte de la encuesta se evalúa el nivel de satisfacción con aspectos generales de la asignatura.

- La segunda parte pregunta sobre la importancia de aspectos relacionados con la preparación de la asignatura.

- En la tercera parte se analiza la metodología docente y la evaluación continua.

- La cuarta parte tiene por objetivo analizar aspectos específicos de la evaluación continua en cada universidad.

En ambas asignaturas (y por tanto en ambas universidades) el cuestionario tiene las mismas preguntas. Sólo hay diferencias en dos aspectos para ajustarlo a las características particulares de cada asignatura. Además, se incluyó una última pregunta abierta para que los estudiantes pudieran escribir aquello que considerasen importante para mejorar la asignatura en futuros cursos.

En la Universidad de Alicante, 33 de los 48 alumnos matriculados en la asignatura (69\%) participaron en este estudio -aquellos que estaban en clase en los días que se pasó la encuesta. El cuestionario se realizó la última semana del cuatrimestre. Los estudiantes que no la completaron fueron algunos ERASMUS que ya habían dejado España aquella semana y aquellos alumnos que no habían asistido a clase regularmente.

En la Universitat de Barcelona, 69 de los 130 alumnos matriculados (53\%) respondieron la encuesta. Fue administrada en la misma semana, después de realizar la última actividad evaluable. Los alumnos que no la completaron fueron los que no seguían la evaluación continua o aquellos que consideraron que ya tenían nota suficiente y no realizaron la última actividad.

Para el segundo objetivo, en ambas asignaturas, uno de los estudios de casos desarrollado y presentado por los estudiantes ha sido un código de conducta para la asignatura. Para hacer el ejercicio, se proporcionó un código ético de una empresa como ejemplo, el mismo en ambas universidades, se realizó en equipo, y se dieron 3 semanas de tiempo para la propuesta. Basado en las propuestas de los alumnos, se ha diseñado el primer documento de código de conducta para la asignatura de Gestión de la Calidad. En esta etapa de la actividad, el alumnado ha diseñado el código, pero no se ha trabajado en cómo implantarlo y mantenerlo, que se realizará como actividades futuras una vez el código de la asignatura sea definitivo, o los cambios aplicados sean menores (primera parte de la norma ISO 10001).

\section{RESULTADOS}

\subsection{Opiniones del alumnado sobre la evaluación continua}

Con relación al primer conjunto de preguntas sobre el nivel de satisfacción del alumnado con la asignatura Gestión de la calidad en ambas universidades (Tabla 1), los resultados mostraron que en la Universidad de Alicante el alumnado está muy satisfecho con el sistema de evaluación y con el clima creado en la clase, seguido de la metodología y el contenido de la materia. 
Merce Bernardo, Juan José Tarí Propuesta de código ético para la asignatura de Gestión de la Calidad

TABLA 1. SATISFACCIÓN DEL ALUMNAdo CON VARIOS ASPECTOS DE LA MATERIA

\begin{tabular}{|l|c|c|c|c|c|c|c|c|c|}
\hline & \multicolumn{3}{|l|}{ Universidad de Alicante } & \multicolumn{3}{|l|}{ Universitat de Barcelona } \\
\cline { 2 - 12 } & Mín & Máx & Media & Desv. & Mín & Máx & Media & Desv. \\
\hline Los contenidos de la asignatura & 2 & 5 & 3,91 & 0,76 & 1 & 5 & 3,33 & 1,02 \\
\hline La metodología de la asignatura & 2 & 5 & 3,91 & 0,91 & 1 & 5 & 3,03 & 1,04 \\
\hline El sistema de evaluación de la asignatura & 2 & 5 & 4,42 & 0,75 & 1 & 5 & 3,26 & 1,21 \\
\hline El clima en el aula & 2 & 5 & 4,21 & 0,92 & 1 & 5 & 2,96 & 1,29 \\
\hline
\end{tabular}

Los resultados de la Universitat de Barcelona también muestran resultados positivos y similares. El alumnado está más satisfecho con el contenido de la asignatura y el sistema de evaluación siendo el clima de la clase el aspecto menos valorado aunque con una puntuación elevada (próxima a 3). Una explicación es que en esta universidad en el grupo de la tarde la docencia era en inglés y había algunos alumnos que les ha costado más seguir la clase.

Respecto a la opinión del alumnado sobre el grado en que ciertas actividades le ayudan a preparar la asignatura (Tabla 2), los resultados indicaron que el alumnado de ambas universidades considera bastante o muy útil el uso del material facilitado por el profesor a través del campus virtual y los estudios de casos realizados en clase y en equipo. Estos aspectos fueron los más útiles para preparar la asignatura, seguidos por la asistencia. Por su parte, la valoración más baja fue para el uso de tutorías, aunque este resultado es debido a que, como muchos nos indicaron, realmente no las utilizaron.

TABLA 2. ActividAdes QUE AYUdAN Al ALUMNADO A PREPARAR LA MATERIA

\begin{tabular}{|c|c|c|c|c|c|c|c|c|}
\hline & \multicolumn{4}{|c|}{$\begin{array}{l}\text { Universidad de } \\
\text { Alicante }\end{array}$} & \multicolumn{4}{|c|}{$\begin{array}{l}\text { Universitat de } \\
\text { Barcelona }\end{array}$} \\
\hline & Mín. & Máx. & Media & Desv. & Mín. & Máx. & Media & Desv. \\
\hline La asistencia a las clases & 2 & 5 & 3,82 & 1,01 & 1 & 5 & 3,39 & 1,23 \\
\hline $\begin{array}{l}\text { Los materiales facilitados por el profesor en clase } \\
\text { o a través del campus virtual }\end{array}$ & 2 & 5 & 4,30 & 0,84 & 1 & 5 & 3,75 & 0,91 \\
\hline $\begin{array}{l}\text { La realización y corrección de los trabajos } \\
\text { prácticos en equipo y en las clases }\end{array}$ & 2 & 5 & 4,12 & 0,81 & 2 & 5 & 3,48 & 1,12 \\
\hline Las tutorías presenciales y/o virtuales & 1 & 5 & 2,97 & 1,32 & 1 & 5 & 2,98 & 1,26 \\
\hline
\end{tabular}

Finalmente, respecto a las actividades de evaluación continua llevadas a cabo en cada universidad, la Tabla 4 nos indica que los estudiantes de la Universidad de Alicante consideran los dos test realizados sobre la asignatura (el primero de los tres primeros temas y el segundo de los tres siguientes) y la presentación y entrega del trabajo final claves para la evaluación continua. 
Tabla 3. Actividades relacionadas Con la metodología y el sistema de eVAluación

\begin{tabular}{|c|c|c|c|c|c|c|c|c|}
\hline & \multicolumn{4}{|c|}{$\begin{array}{l}\text { Universidad de } \\
\text { Alicante }\end{array}$} & \multicolumn{4}{|c|}{$\begin{array}{l}\text { Universitat de } \\
\text { Barcelona }\end{array}$} \\
\hline & Mín & Máx. & Media & Desv. & Mín. & Máx. & Media & Desv \\
\hline $\begin{array}{l}\text { La explicación teórica por parte del profesor es } \\
\text { necesaria }\end{array}$ & 2 & 5 & 3,82 & 0,98 & 1 & 5 & 3,61 & 1,19 \\
\hline $\begin{array}{l}\text { Las transparencias de cada tema ayudan a seguir } \\
\text { la materia }\end{array}$ & 1 & 5 & 3,94 & 0,93 & 1 & 5 & 3,71 & 1,04 \\
\hline $\begin{array}{l}\text { El trabajo en equipo en la clase práctica (para } \\
\text { resolver los casos de cada tema) me parecen } \\
\text { importantes para mejorar nuestros conocimientos }\end{array}$ & 3 & 5 & 4,12 & 0,73 & 1 & 5 & 3,51 & 1,24 \\
\hline $\begin{array}{l}\text { El proceso de evaluación continua ( } 2 \text { exámenes } \\
\text { parciales, } 2 \text { trabajos cortos a entregar, trabajo } \\
\text { final) fomenta la asimilación de los contenidos }\end{array}$ & 2 & 5 & 4,48 & 0,75 & - & - & - & - \\
\hline $\begin{array}{l}\text { El proceso de evaluación continua (examen test, } \\
\text { casos en clase y trabajo autónomo) fomenta la } \\
\text { asimilación de los contenidos }\end{array}$ & - & - & - & - & 1 & 5 & 3,67 & 1,08 \\
\hline $\begin{array}{l}\text { La evaluación continua permite seguir mejor el } \\
\text { ritmo de la asignatura }\end{array}$ & 1 & 5 & 4,48 & 0,87 & 1 & 5 & 3,90 & 1,07 \\
\hline $\begin{array}{l}\text { La evaluación continua ayuda a aprender más } \\
\text { que con el sistema tradicional basado sólo en un } \\
\text { examen final }\end{array}$ & 1 & 5 & 4,36 & 1,11 & 1 & 5 & 3,99 & 1,24 \\
\hline $\begin{array}{l}\text { El material on-line sobre cómo redactar un } \\
\text { procedimiento y cómo hacer un diagrama causa- } \\
\text { efecto me parece útil para trabajar desde casa }\end{array}$ & 3 & 5 & 4,12 & 0,78 & - & - & - & - \\
\hline $\begin{array}{l}\text { Las actividades no obligatorias (debate en clase, } \\
\text { resolución de casos, etc.) son útiles para aplicar } \\
\text { fácilmente la teoría }\end{array}$ & - & - & - & - & 1 & 5 & 3,26 & 1,17 \\
\hline $\begin{array}{l}\text { Las actividades no obligatorias deberían ser } \\
\text { obligatorias }\end{array}$ & - & - & - & - & 1 & 5 & 3,03 & 1,44 \\
\hline $\begin{array}{l}\text { Las actividades no evaluables (debate en clase, } \\
\text { resolución de casos en clase, etc.) son útiles para } \\
\text { aplicar la teoría de forma sencilla }\end{array}$ & 2 & 5 & 3,88 & 0,96 & - & - & - & - \\
\hline
\end{tabular}

Respecto a los dos casos cortos, aunque también son importantes para evaluar otros aspectos dentro del proceso de evaluación continua, su valoración no fue tan alta como en las dos anteriores actividades. Este resultado puede deberse a que son dos casos cortos que realmente requieren menos trabajo y tiempo del alumno y a su vez su puntuación en la nota final es más baja que en las otras dos actividades. 
Tabla 4. Actividades de evaluación continua en la Universidad de Alicante

\begin{tabular}{|l|c|c|c|c|}
\hline & Mínimo & Máximo & Media & Desv. \\
\hline Los 2 exámenes parciales tipo test & 1 & 5 & 4,06 & 0,82 \\
\hline La realización de los 2 trabajos cortos & 1 & 5 & 3,76 & 1,00 \\
\hline La realización y entrega del trabajo final & 2 & 5 & 4,24 & 0,79 \\
\hline
\end{tabular}

La Tabla 5 muestra que para el alumnado de la Universitat de Barcelona los aspectos más valorados son los exámenes realizados después de cada unidad seguida del trabajo en clase y finalmente el trabajo autónomo. Los resultados muestran que los estudiantes prefieren ser evaluados en clase que tener que trabajar la materia fuera de ella. Se puede interpretar por la carga de trabajo durante el grado, hecho que se podría resolver con una mayor coordinación y aprovechamiento de sinergias entre las diferentes asignaturas del grado.

tabla 5. Actividades de evaluación continua en la Universitat de Barcelona

\begin{tabular}{|l|c|c|c|c|}
\hline & Mínimo & Máximo & Media & Desv. \\
\hline Exámenes test después de cada tema & 2 & 5 & 3,87 & 0,68 \\
\hline $\begin{array}{l}\text { Resolución de casos en clase (causa-efecto, auditoria, etc.) } \\
\begin{array}{l}\text { Resolución de casos como trabajo autónomo (código } \\
\text { conducta, etc.) }\end{array}\end{array}$ & 1 & 5 & 3,52 & 0,99 \\
\hline
\end{tabular}

A partir de estos resultados se observa que hay mínimas diferencias entre el alumnado de una y otra universidad basadas en una valoración distinta en algunos aspectos. Por ejemplo, en la Universidad de Alicante el aspecto metodológico que menos útil les pareció son las actividades no evaluables y en la Universitat de Barcelona las actividades no obligatorias, o que el clima en la clase es más valorado por los alumnos de la Universidad de Alicante que por los de la Universitat de Barcelona. No obstante, en general, predominan las semejanzas entre las opiniones del alumnado de ambas universidades. En este sentido, el alumnado de ambas universidades está satisfecho con el contenido, la metodología y el sistema de evaluación de las asignaturas. En ambos casos consideran que los materiales facilitados por el profesor y el trabajo hecho en equipo en clase para resolver los casos son básicos para su aprendizaje así como que el uso de las tutorías suele ser igual que con el sistema tradicional. Las tutorías es el aspecto menos valorado aunque parece ser que esto es debido a la no utilización de las mismas.

De igual forma, debemos destacar que respecto a la metodología de las clases, los aspectos más valorados son: el proceso de evaluación continua porque fomenta la asimilación de contendidos, la evaluación continua permite seguir mejor el ritmo de la asignatura y la evaluación continua ayuda a aprender más que con el sistema tradicional.

Esto significa que la evaluación continua es preferida al sistema tradicional basada en el examen final porque facilita la asimilación de los contenidos, permite seguir el ritmo 
de la asignatura al estudiar de una manera más continua y como consecuencia, como nos indicaron, ayuda a aprender más.

Por su parte, lo que menos valoran son las actividades no evaluables o no obligatorias. Consideramos que son necesarias para el aprendizaje pero también parece claro que si queremos aumentar el interés del alumnado por estas actividades hay que evaluarlas.

\subsection{Código de CONDUCTA DE LA ASIGNATURA}

El segundo objetivo de este artículo es proponer un documento de código de conducta para la asignatura de gestión de la calidad. Se comentan brevemente los resultados de cada universidad para presentar el código de conducta propuesto para la asignatura de gestión de la calidad.

Los alumnos de la Universidad de Alicante consideran importante el cumplimiento de las normas, la comunicación entre profesor y alumnos, el respeto mutuo y el cuidado de las instalaciones, el sistema de evaluación justo y las sanciones por mal comportamiento.

Los alumnos de la Universitat de Barcelona dan importancia al respecto y no discriminación de ningún tipo y por ningún motivo, el cumplimiento de las normas, el comportamiento adecuado tanto en clase como en la elaboración de los ejercicios y en la no corrupción.

Un análisis de los códigos éticos presentados por los equipos de alumnos nos permite proponer un primer ejemplo de código ético (Tabla 6). Está compuesto por 11 principios y aglutina los principios más destacados por los estudiantes en ambas universidades.

\section{CONCLUSIONES}

El objetivo de este artículo es doble. Primero, proponer y analizar una encuesta para medir la satisfacción de los estudiantes con la metodología de evaluación continua. Segundo, proponer un código de conducta de la asignatura de gestión de la calidad.

Para lograr el primer objetivo se han analizado los resultados de una encuesta que se pasó en dos universidades. Respondieron un total de 102 estudiantes, 33 estudiantes de la Universidad de Alicante y 69 de la Universitat de Barcelona.

Los resultados muestran algunas diferencias leves. Por ejemplo, en la Universidad de Alicante los estudiantes valoran positivamente el clima en clase mientras que en la Universitat de Barcelona, valoran más el contenido de la asignatura. No obstante, claramente destacan las similitudes entre ambas universidades. Por ejemplo, los aspectos más valorados en cada asignatura y universidad son la aplicación de la metodología de evaluación continua (por ejemplo, a través de exámenes test) que es preferida al examen final porque el alumnado puede llevar la asignatura más preparada, les permite aprender más que con el método tradicional y siguen mejor el ritmo de la asignatura. Otros aspectos comunes entre ambas universidades son:

- Relacionados con la asignatura, los estudiantes valoran positivamente el sistema de evaluación.

- Respecto a la evaluación continua, ayuda a preparar la asignatura, y los estudiantes están satisfechos con los materiales proporcionados y los casos hechos en clase. La evaluación continua, utilizando entre otras actividades exámenes tipo test, es adecuada. 
tabla 6. Propuesta de Código de Conducta para la asignatura de Gestión de la Calidad

\begin{tabular}{|c|c|}
\hline Principio & Descripción \\
\hline $\begin{array}{l}\text { Cumplimiento de } \\
\text { la normativa }\end{array}$ & $\begin{array}{l}\text { - Cumplimiento de normativa: normas estatuto universidad, normas } \\
\text { estatuto estudiante, normas de la asignatura }\end{array}$ \\
\hline Comunicación & $\begin{array}{l}\text { - El profesor comunica los objetivos, metodología, sistema de evaluación, } \\
\text { etc., al inicio del curso académico y los hace públicos } \\
\text { - El profesor informa de las normas y sistema de evaluación de los trabajos y } \\
\text { los hace público }\end{array}$ \\
\hline Evaluación & $\begin{array}{l}\text { - El/la alumno/a entrega los trabajos en los plazos establecidos } \\
\text { - El profesor evalúa de manera justa y según criterios claros y objetivos } \\
\text { - El alumno/a mantiene una actitud honesta en el examen (no copiar) } \\
\text { - Debe permitir un seguimiento adecuado de la asignatura }\end{array}$ \\
\hline Comportamiento & $\begin{array}{l}\text { - Respeto mutuo entre profesorado y alumnos } \\
\text { - Profesor debe asegurarse que el conocimiento es adquirido por igual por } \\
\text { todos los alumnos, motivar la participación, etc. } \\
\text { - Los alumnos deberán comportarse educadamente en clase, prestando } \\
\text { atención, no utilizando los dispositivos electrónicos si no es necesario, } \\
\text { siendo puntuales, participando en clase, etc. } \\
\text { - Respeto mobiliario, instalaciones de la universidad, etc. } \\
\text { - Respeto horas tutoría } \\
\text { - Contestar al alumno una tutoría on-line antes de } 72 \text { horas } \\
\text { Respeto diversidad alumnos, no discriminar }\end{array}$ \\
\hline $\begin{array}{l}\text { Otras actividades } \\
\text { externas }\end{array}$ & $\begin{array}{l}\text { - El profesorado y el alumnado podrán realizar actividades relacionadas con } \\
\text { la enseñanza (impartir o recibir clases de todo tipo) siempre que no } \\
\text { interfiera en la actividad requerida para el desarrollo de la asignatura }\end{array}$ \\
\hline Conflicto de intereses & $\begin{array}{l}\text { - No anteponer los intereses personales a los intereses del conjunto. } \\
\text { - Comunicar al profesor o representante de los alumnos el posible conflicto } \\
\text { de intereses }\end{array}$ \\
\hline $\begin{array}{l}\text { Información } \\
\text { confidencial } \\
\text { (protección de } \\
\text { la información) }\end{array}$ & $\begin{array}{l}\text { - Cumplimiento normativa sobre información alumnos (notas, exámenes, } \\
\text { datos personales, etc.): Ley de protección de datos } \\
\text { - Está prohibido el plagio (copia trabajos cursos anteriores, Internet, etc.) }\end{array}$ \\
\hline Soborno y corrupción & $\begin{array}{l}\text { - Repudiar toda forma de soborno y corrupción } \\
\text { - Si se observan conductas ilegales o que violen las normas anteriormente } \\
\text { establecidas se deberá comunicar al responsable de la clase para } \\
\text { encontrar una solución al conflicto lo más rápido posible y así poder evitar } \\
\text { futuras incidencias } \\
\text { - Si es necesario, se deberá comunicar a los superiores para asegurar que el } \\
\text { comportamiento sea el adecuado }\end{array}$ \\
\hline $\begin{array}{l}\text { Sanciones en caso de } \\
\text { incumplimiento }\end{array}$ & $\begin{array}{l}\text { - Si se incumple este código, se sancionará en función del principio y del } \\
\text { grado de incumplimiento } \\
\text { - Copiar en los exámenes o los ejercicios es sancionable }\end{array}$ \\
\hline $\begin{array}{l}\text { Otros grupos de } \\
\text { interés }\end{array}$ & $\begin{array}{l}\text { - Las relaciones de parentesco y/o amistad no pueden interferir en el } \\
\text { desarrollo de las clases ni en la evaluación. Tampoco podrá repercutir en } \\
\text { un trato preferencial en ninguna de las relaciones que se pueden dar en } \\
\text { clase }\end{array}$ \\
\hline
\end{tabular}


Comunidad

universitaria
- Facilitar instalaciones y material, mantener en buenas condiciones las instalaciones de la universidad, ofrecer servicios de apoyo al estudiante, disponer de personal de apoyo a las clases, etc.

Respecto al código de conducta, los resultados de las encuestas muestran que el caso del código de conducta es útil para los alumnos. No obstante, no obtiene la valoración más alta porque como ejercicio que se evalúa, su ponderación de la nota final es mucho menor que por ejemplo el trabajo final. Además, aunque el alumnado ha utilizado como guía el ejemplo de una empresa, es de destacar que consideran importantes aspectos como el plagio e incluso sancionar determinados comportamientos poco éticos como, por ejemplo, copiar en los exámenes. A partir de los códigos entregados por los equipos de alumnos, se ha propuesto un documento para la asignatura que puede ser fácilmente aplicable a otras asignaturas. Algunos principios aún deben desarrollarse con más claridad, pero la estructura y base del código pueden servir como ejemplo. Los principales principios se centran en el respeto y comportamiento, la evaluación de la asignatura y el cumplimiento de las normas y del propio código ético.

Las similitudes y diferencias pueden ayudar a mejorar las actividades en el siguiente curso académico en la asignatura de gestión de la calidad y otras asignaturas relacionadas. Finalmente, la comparación entre las dos universidades en cuanto a la opinión de los estudiantes y el código de conducta permitirán diseñar una metodología específica para la asignatura y proponer guías para próximos cursos de gestión de empresas.

Las principales limitaciones son el número de participantes que respondieron el cuestionario y el momento de recoger los datos. Para una futura investigación se podrían recoger datos adicionales para comparar la evolución de la evaluación continua y analizar la aplicación y cumplimiento con el código de conducta, así como, para compararlas mejor, se podrían igualar más las metodologías y evaluaciones en ambas universidades.

\section{BIBLIOGRAFÍA}

Adam, A.M. y RaChMAN-MOORE, D. [2004]: The Methods Used to Implement an Ethical Code of Conduct and Employee Attitudes, Journal of Business Ethics, núm. 54: 225-244.

BRAXTON, J.M. Y BRAY, N.J. [2012]: Introduction: The Importance of Codes of Conduct for Academia, New Directions for Higher Education, núm. 160: 1-4.

CAstilla Polo, F. [2011]: Calidad docente en el ámbito universitario: Un estudio comparativo de las universidades andaluzas, Revista de Educación en Contabilidad, Finanzas y Administración de Empresas, núm. 2: 157 - 172.

Claver Cortés, E., lópez Gamero, M.D., Marco lajara, B., Molina Azorín, J.F., Pereira Moliner, J., Pertusa Ortega, E., Quer Ramón, D. y Zaragoza Sáez, P.C. [2007]: La estimación del esfuerzo y tiempo para el aprendizaje de Dirección Estratégica: un análisis cuantitativo y cualitativo. En M.A. MARTíNEZ Y V. CARRASCO (Eds.), La multidimensionalidad de la educación universitaria. Redes de investigación docente- Espacio Europeo de Educación Superior, vol. I: 41-70. Marfil, Alcoy.

De Juana-Espinosa, S., Valdés-Conca, J., Fernández-SÁnChez, J.A., GonzÁlez-RAmírez, M.R., MANResa-Marhuenda, E., SAbater Sánchez, V., Andreu Guerrero, R., Tarí Guilló, J.J., Y RIENDA GARCÍA, L. [2010]: Assessing the students' perception about learning competences in a management course. International Conference on Education and New Learning Technologies (EDULEARN10). Barcelona, Julio. 
Delgado, A.M. y Oliver, R. [2006]: La Evaluación Continua en un nuevo Escenario Docente, Revista de Universidad y Sociedad del Conocimiento, vol.3, núm.1, http://www.uoc.edu/rusc/3/1/dt/esp/delgado_oliver.pdf.

EFQM [2012]: EFQM Model for Business Excellence: Company Guidelines, European Foundation for Quality Management, Brussels, Belgium.

Garandillas Iglesias, M., Somohano RodríGuez, F. M. y Martínez García, F.J. [2011]: Innovación y evaluación en el marco del Espacio Europeo de Educación Superior: Una experiencia piloto utilizando herramientas Web 2.0, Revista de Educación en Contabilidad, Finanzas y Administración de Empresas, núm. 2: $85-111$.

GONZÁlEZ, C. Y RAMOS, M.P. [2007]: Aplicación de un sistema de evaluación continua a alumnos de $5^{\circ}$ de Farmacia, en el proceso de adaptación al Espacio Europeo de Educación Superior, Revista d'educació superior en Farmàcia, núm. 1: 1-10.

González Pareja, A., Calderón Montero, S., Torrico González, A. y Rodríguez Díaz, B. [2004]: Una experiencia en la enseñanza-aprendizaje de Investigación Operativa, XII Jornadas de la Asociación Española de Profesores Universitarios de Matemáticas para la Economía y la Empresa, Murcia, septiembre. http://www.uv.es/asepuma/XIl/comunica/gonzalez_calderon_torrico_rodrigue Z.pdf.

Gras García, L., Jiménez Millagón, A., Maestre Pérez, S.E., Mora Pastor, J., Prats Moya, M.S. Y TODOLí TORRO, J.L. [2006]: Desarrollo de metodologías docentes basadas en el trabajo colaborativo y actividades no presenciales en laboratorios integrados de la titulación en química. En M.J. Frau Y N. Sauleda (Eds.). La reconfiguración curricular en el escenario universitario. Redes de Investigación Docente en el Espacio Europeo de Educación Superior. II:161-187. Marfil, Alcoy.

ISO [2004]: ISO 10002 Quality management- Customer satisfaction- Guidelines for complaints handling in organizations, International Organization for Standardization, Geneva, Switzerland.

ISO [2005]: ISO 9000 Quality management systems - Fundamentals and vocabulary, International Organization for Standardization, Geneva, Suiza.

ISO [2007A]: ISO 10001 Quality management- Customer satisfaction- Guidelines for codes of conduct for organizations, International Organization for Standardization, Geneva, Switzerland.

ISO [2007B]: ISO 10003 Quality management- Customer satisfaction- Guidelines for dispute resolution external to organizations, International Organization for Standardization, Geneva, Switzerland.

ISO [2009]: ISO/CD TS 10004 Quality management- Customer satisfaction- Guidelines for monitoring and measuring, International Organization for Standardization, Geneva, Switzerland.

Martínez, M.A. y SauledA, N. [2004]: Redes para investigar el currículo. Diseño del aprendizaje en el Espacio Europeo de la Educación Superior (EEES). En Bernabeu, J.G. y Sauleda, N., Investigar el Espacio Europeo de Educación Superior, Vicerrectorado de Convergencia Europea y Calidad, Instituto de Ciencias de la Educación, Alicante: Universidad de alicante.

MARTínez, M.A. y SAULEDA, N. [2005]: La investigación basada en el diseño y el diseño del crédito europeo. En Martínez y Carrasco (Eds.) Investigar en diseño curricular. Redes de docencia en el Espacio Europeo de Educación Superior: 7-22. Marfil, Alcoy.

MICHAVILA, F. y PÉREZ, F. [2007]: Análisis de las estrategias de adaptación de la oferta educativa de la Universidad de Alicante al Espacio Europeo de Educación Superior. Marfil, Alcoy. 
MINISTERIO DE EDUCACIÓN y CIENCIA (MEC) [2006]: Propuestas para la renovación de las metodologías educativas en la universidad, http://www.catedraunesco.es/archivos/metodologias.pdf

Montoro-SánCHEZ, M.A., MORA-VAlentín, E.M. y ORtiz-DE-URBINA-CRIADO, M. [2012]: Análisis de las competencias adquiridas en los estudios de Dirección de Empresas y su grado de aplicación en las prácticas en empresas, Revista Complutense de Educación, núm.1: 241-263.

Pascual Ezama, D., Camacho Miñano, M.M., Urquía Grande, E. y Müller, A. [2011]: ¿̇Son los nuevos criterios de evaluación en el marco del EEES adecuados para valorar el rendimiento académico de los alumnos? Experiencia en Contabilidad Financiera, Revista de Educación en Contabilidad, Finanzas y Administración de Empresas, núm. 2: $67-83$.

PlazA, F.J., GÓMEZ, E., LÓPEZ, A.C. y FERnÁNDEZ, A.A. [2010]: Reflexiones en torno a una experiencia de Evaluación Continua en el EEES, Revista Docencia e Investigación, núm. 20: 11-32.

RUIZ, J.R. Y CASTAÑO, S. [2008]: La universidad española ante el resto del EEES, Docencia e Investigación, Vol. 8 (versión digital), año XXXIII, enero/diciembre.

http://www.uclm.es/varios/revistas/docenciaeinvestigacion/pdf/numero8/Jose_Reyes_ Ruiz_Gallardo.doc.

SABATER, V., TARÍ, J.J. y RIENDA, L. [2012]: Students' preferences regarding methodology and satisfaction on continuous assessment models, International Conference on Education and New Learning Technologies (EDULEARN 2012), Barcelona, 24 de Julio.

Sethl, S.P., Veral, E.A., Shapiro, H.J. y Emellanova, O. [2011]: Mattel, Inc.: Global Manufacturing Principles (GMP) - A Life-Cycle Analysis of a Company-Based Code of Conduct in the Toy Industry, Journal of Business Ethics, núm. 99: 483517.

UCA [2005]: Código Peñalver - Código ético de la Universidad de Cádiz, Universidad de Cádiz. 